\title{
CWR track vibration characteristics varying with the change of supporting condition
}

\author{
L. Li \& Y. Luo \\ Railway and Urban Mass Transit Research Institute, \\ Tongji University, China
}

\begin{abstract}
Stress contained in rails, mainly due to thermally-induced expansion and contraction, results in large longitudinal loads which lead to broken rails and track buckling. According to the beam bending theory of structural dynamics with a consideration of the influence of axial load, a test method to measure the longitudinal force in CWR is introduced. This paper studied the elements which will affect the results used in this method such as the total calculation and measurement length of rail, unsupported length of rail. Standard relation curve between longitudinal force and first order frequency was provided. To the actual continuous welded rail, longitudinal force can be calculated according to the standard relation curve after the vibration response frequency of rail in some state is measured. This method would help to evaluate and measure the force in rail caused by temperature change.

Keywords: continuous welded rail (CWR), buckling, stability, natural frequency, vibration characteristics.
\end{abstract}

\section{Introduction}

Continuous welded rail (CWR) is widely used in modern railways because it replaces jointed track for the better economics of maintenance and ride comfort. If the geometry of straight track is in good condition (so do the fasteners, sleepers and ballast), the rail will not buckle. However, due to the manufactured error or other reasons, the slight loss of straightness will result in the track buckling. If the ballast shoulders are in poor condition, the lateral stability of the track is low and the buckle will be horizontal. If the ballast shoulders are good, 
the buckle can be vertical so that sleepers are lifted out of the ballast resulting in the track hanging in the air totally unsupported.

Traditionally the way to measure the stress in CWR has been to cut the rail. Although the accuracy of this method is high, it is disruptive and expensive. Theoretical prediction of thermal stress, based on the measurement of rail temperature, is difficult due to numerous, usually unknown factors describing track structure and its displacements. Researchers in the worldwide have been studying on how to measure the rail stress and the stability of CWR [1-5]. Rail stress can be measured by rail uplift, application of strain sensors or ultrasonic technique. They have significant limitations.

Theoretically vibration characteristics of CWR vary regularly with the different longitudinal force. If the rule can be obtained within the certain rail, the information of real rail stress can be gotten according to analyzing its vibration parameters. The objective of this study is to develop a new, comprehensive method to get the relationship between the longitudinal force and the vibration characteristics of CWR.

\section{Mechanical theory}

Being a kind of complex structure system, the stability of CWR track structure is decided by a multiple factors which work together. The theoretical long-term study and large number of experiments show that stability of CWR is related by the temperature force, rail initial irregularities, the track horizontal resistance, rail structural frame stiffness and fasteners resistance moment.

According to the structural dynamics theory, the axial force and any loads which tend to cause the buckling, probably produce great effect on structure stiffness [6]. Component force which is parallel with original axis of member will cause additional load component $\vec{f}_{G}$ along (and point to) node displacement direction.

When the temperature rises, the deflection will be increased. Then the dynamic balance for component expression is:

$$
\begin{gathered}
\vec{f}_{I}+\vec{f}_{K}+\vec{f}_{D}-\vec{f}_{G}=\vec{P}(t) \\
\vec{f}_{G}=k_{G} \vec{v}
\end{gathered}
$$

In which geometric stiffness influence coefficient $k_{G_{i j}}$ is defined:

$k_{G_{i j}}=$ the force corresponding to the coordinate $i$ caused by the unit displacement at coordinate $j$ and axial force component in structure. Here $k_{G}$ is called the geometry stiffness matrix of structure.

Structural dynamic balance equation is:

$$
m \ddot{\vec{v}}+c \dot{\vec{v}}+k \vec{v}-k_{G} \vec{v}=\vec{P}(t)
$$


Because both the elastic and geometric stiffness multiply with displacement vector, the combination stiffness effect can be used as a single symbol. Then the above equation can be written as follows:

$$
m \ddot{\vec{v}}+c \dot{\vec{v}}+\bar{k} \vec{v}=\vec{P}(t)
$$

In which, $\bar{k}=k-k_{G}$ is called the combination stiffness matrix, it contains both elastic and geometric effects.

Single-degree-of-freedom system of damping vibration frequency is

$$
\omega_{D}=\omega \sqrt{1-\zeta^{2}}=\sqrt{\frac{\bar{k}}{m}} \sqrt{1-\zeta^{2}}
$$

If we get a rail vibration response curve (under certain axial force) as in Figure 1.

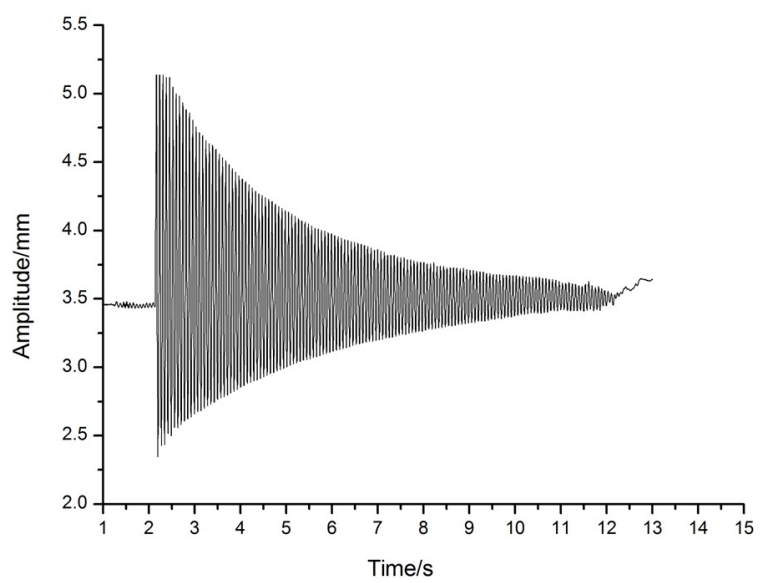

Figure 1: Horizontal vibration response in situ of rail with unsupported length of $5.3 \mathrm{~m}$.

From the curve, there are such parameters can be obtained, such as logarithmic decrement $\delta=\ln \frac{v_{n}}{v_{n+1}}$, damping ratio $\zeta=\frac{\delta}{2 \pi}$ and damping coefficient $c=\zeta c_{r}=\zeta 2 m \bar{\omega}$.

It shows there is some relationship between the system vibration frequency, damping coefficient and axial force. In fact, under the role of axial force (compression is positive), transverse vibration damping of beam structure will become bigger with the axial load increases. While transverse vibration frequencies will decrease with the axial load increase. 


\section{Rail vibration analysis}

\subsection{Rules when temperature rise}

Figure 2 shows the rules of that first frequency when the temperature rises. It can be seen that the first frequency of rail will decrease as the temperature increase. The basic trend of the first vibration mode has two stages: when the degree of temperature rise is small, its variation rule are similar with the rail under axial compression, that is, a linear relationship between them; as the temperature increases, this relationship has the effect of non-linear change, until the track produce instability. The result is affected by the length of rail. When the length of rail is shorter, the data is relatively bigger. The results of the latter two lengths of rail are relative similar.

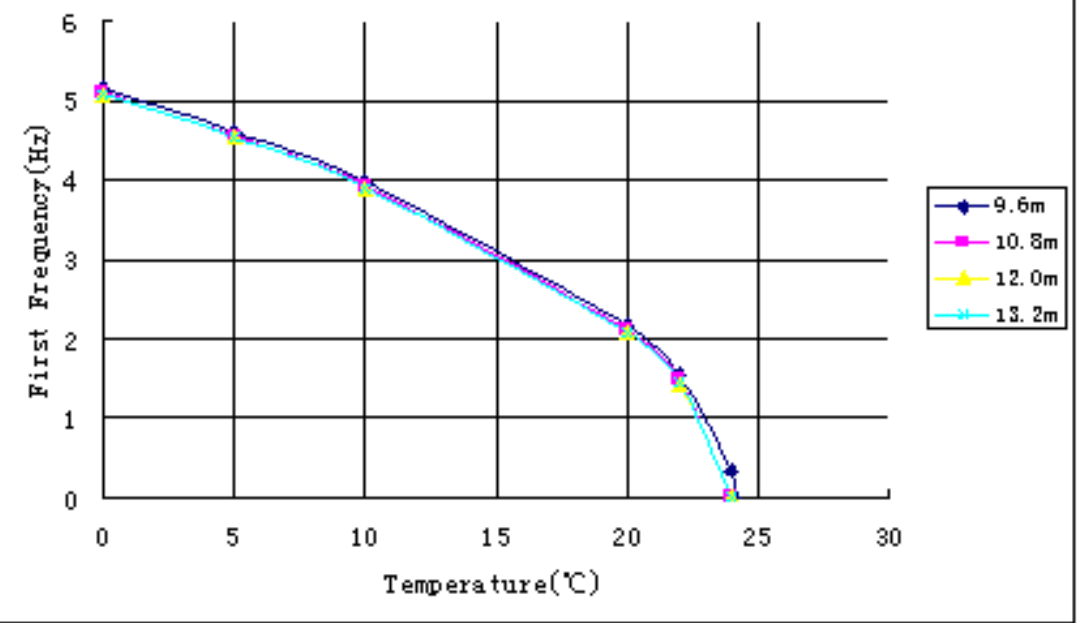

Figure 2: $\quad$ First frequency analysis when temperature rises.

\subsection{Determination of test parameters}

CWR can be simplified as a uniform beam with infinite length under the role of longitudinal force supported by equal distance elastic springs (Figure 3 ). When remove fasteners within a certain length and let this range of rail suspended, it is

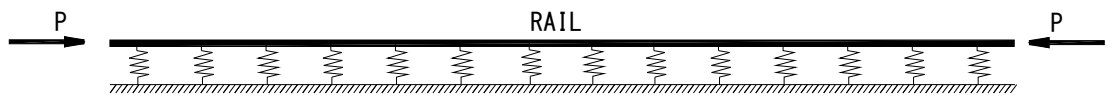

Figure 3: $\quad$ Continuous supported model of CWR track. 
thought the longitudinal force in the whole length of rail is generally the same besides the force is redistributed within that certain length of rail. The model becomes a beam supported by equal distance elastic springs outside of length $L_{0}$ (Figure 4). The main parameters in situ are shown in Tables 1 and 2.

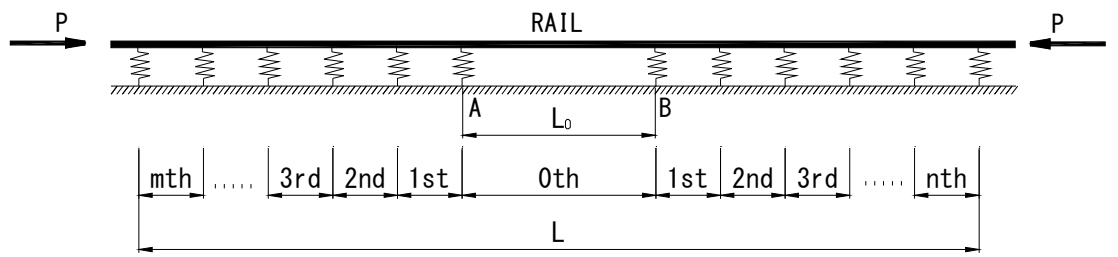

Figure 4: Continuous supported model of CWR track with a length of unsupported.

Table 1: $\quad$ Track parameters.

\begin{tabular}{|c|c|}
\hline Parameters & Reference value \\
\hline Rail size & 60 \\
\hline Tie spacing $[m]$ & 0.6 \\
\hline Track length $[m]$ & 25 \\
\hline Unsupported length $[m]$ & $5.33-13.2$ \\
\hline Vertical stiffness $[N / m m]$ & $\infty, 3.5 \times 10^{7}$ \\
\hline Lateral stiffness $[N / m m]$ & $\infty, 2.6 \times 10^{7}$ \\
\hline Longitudinal stiffness & $\infty, 6.0 \times 10^{7}$ \\
{$[N / m m]$} & \\
\hline
\end{tabular}

Table 2: $\quad$ Rail parameters.

\begin{tabular}{|c|l|l|l|c|}
\hline $\begin{array}{l}\text { Rail } \\
\text { size }\end{array}$ & $\begin{array}{l}\text { Sectional } \\
\text { area } \\
{\left[\mathrm{cm}^{2}\right]}\end{array}$ & $\begin{array}{l}\text { Moment of } \\
\text { inertia } \\
{\left[\mathrm{cm}^{4}\right]}\end{array}$ & $\begin{array}{l}\text { Mass } \\
{[\mathrm{kg} / \mathrm{m}]}\end{array}$ & $\begin{array}{l}\text { Elastic modulus } \\
{\left[\mathrm{N} / \mathrm{cm}^{2}\right]}\end{array}$ \\
\hline 60 & 77.45 & 3217 & 60.64 & $2.1 \times 10^{7}$ \\
\hline
\end{tabular}

\subsubsection{Calculation rail length}

First we need to determine the rail length. So we calculate the different lengths of rail to see how it will affect the results. In these cases $L_{0}$ is same $(9.6 \mathrm{~m})$. 
From Figure 5 it is shown that as the rail length rises, the natural frequencies of the other cases are tending to close except the first case. The error between the third and the forth case is about $0.166 \%$, so from the mode analysis, $12 \mathrm{~m}$ or $13.2 m$ can satisfy demand.

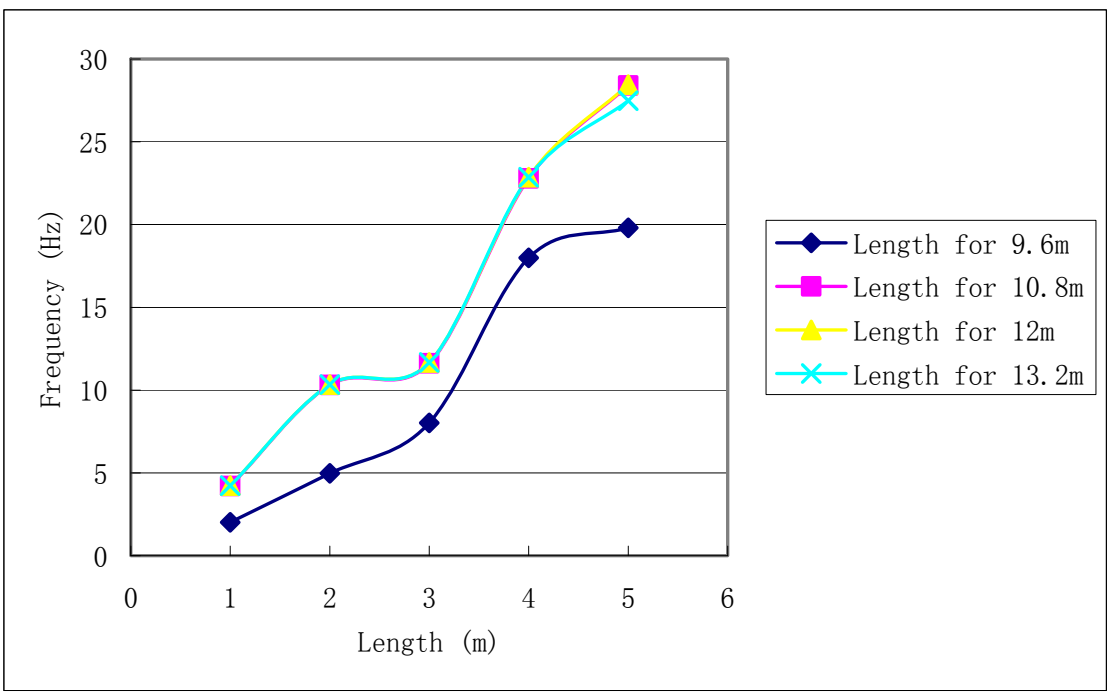

Figure 5: $\quad$ Simply supported rail.

However there is a different between this calculation model and actual situation. In this calculation model it is supposed that the rail is well restrained at the sleepers' position. When there is longitudinal force in rail, the force depends on frictional force to be balance. So the actual length of rail is needed to take this factor into account. Generally the friction of each fastener is about $14 \mathrm{kN}$, so we can get the minimum numbers of fasteners outside of $0.5 L_{0}$ according to the approximate longitudinal force. To a 25-m-length of rail, the maximum temperature which can be endured is $180 \mathrm{kN}$. It means the temperature fluctuate is about 11.6 degrees centigrade.

\subsubsection{Unsupported length}

To determine the unsupported length is another prerequisite element to do the situ-experiment. We have studied the case there is no longitudinal force in the rail firstly. After the rail is excited under the horizontal direction, the vibration response curve can be gotten. The first order of horizontal frequency is in Figure 6.

From Figure 6, it can be seen that when the unsupported length increases, the rail horizontal natural frequency decreases. And the decline trend becomes gently. However, the unsupported length can not be too long, because the rail will be drooped under its gravity. If the deflection is bigger than the space between the rail and sleeper, the rail will touch the sleeper and the rail can not be excited. We adopt $10.4 \mathrm{~m}$ as the unsupported length. 


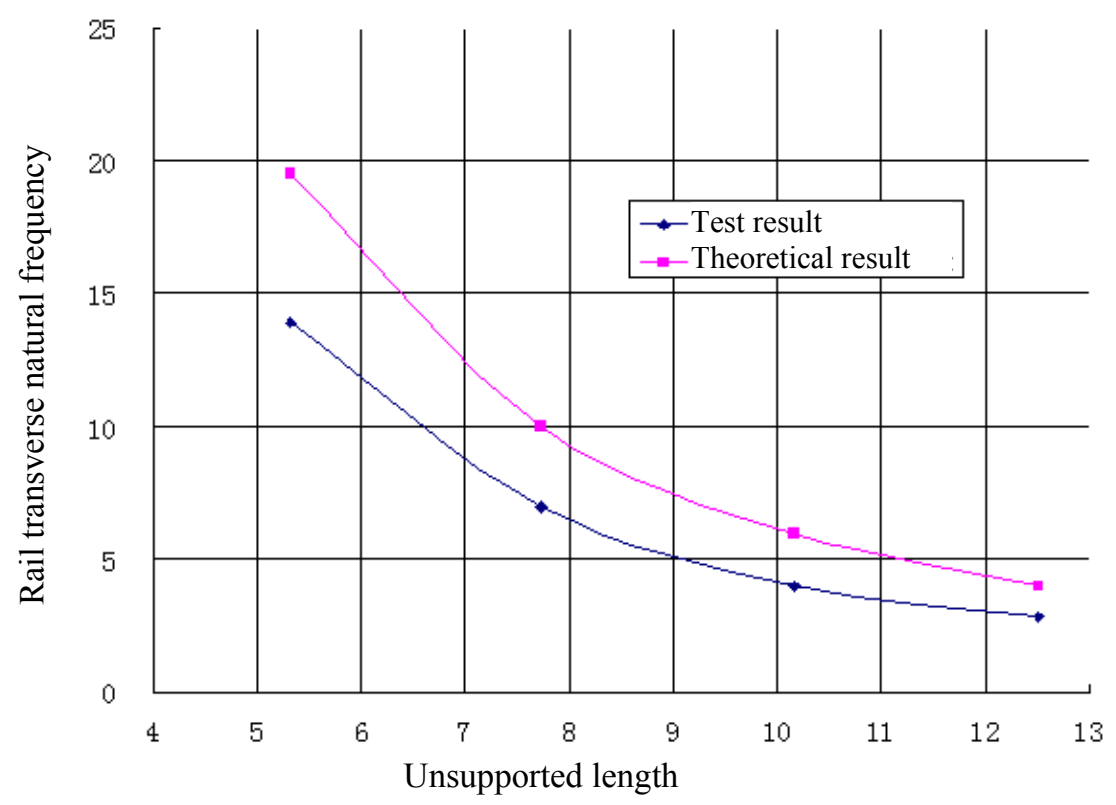

Figure 6: The first order of horizontal frequency.

\subsection{Experiment process}

The experiment process is: firstly build the standard relation curve. Standard relation curves can be made in the laboratory or in situ through the calibration methods to gain. Select a seamless track, rail stress-relieving, and put a load device at one end of rail. Give the rail different compressive load; make the rails produce the required longitudinal force; excite the rail to vibrate; get the vibration frequency. Then as long as vibration response frequency of continuous welded rail in some state is measured, it can be calculated according to the standard relation curve.

Different types of track structure set up the corresponding standard relation curves. Figure 7 is one standard relation curve with $10.4 \mathrm{~m}$ unsupported length.

\section{Conclusions}

Based on the continuous beam theory, the dynamic characteristics of CWR vary with the temperature fluctuate. The object is CWR track with a length of unsupported rail subjected to the longitudinal force due to fluctuation of temperature. The paper introduced a test method to measure the longitudinal force in CWR, built standard relation curve. By compared with standard relation curve after testing the dynamic characteristics of real track, the longitudinal force can be determined. The result would help to evaluated and measure the real temperature in rail. 


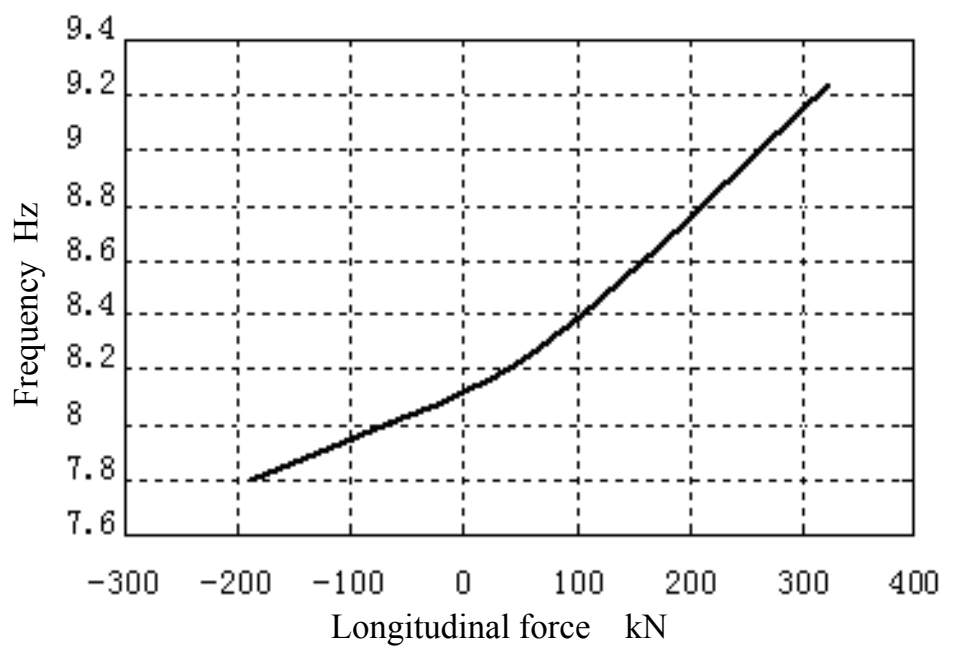

Figure 7: The standard curve between longitudinal force and vibration frequency.

\section{Acknowledgement}

This work was financially supported by the Fundamental Research Funds for the Central Universities (Tongji University), National Science and Technology Support Plan (2009BAG11B02).

\section{References}

[1] Mium S. Lateral track stability: theory and practice in Japan. Lateral Track Stability 1991: Proceeding of a Conference, St. Louis, Missouri: 53-63, 1991.

[2] Kerr A D. An improved analysis for thermal track buckling. Int J Nonlinear Mech, 15(2): pp. 99-114, 1980.

[3] Esveld C, Hengstum L. A. Track stability in tight curves. Rail Int., 12: pp. 15-20, 1988.

[4] Bao Y, Barenberg E J. Three dimension nonlinear stability analysis of tangent CWR track under temperature and mechanical loads. Transportation research record, 1584: pp. 31-40, 1997.

[5] Lim N, Park N, Kang Y. Stability of continuous welded rail track. Computer and Structures, 81: pp. 2219-2236, 2003.

[6] Clough Ray W., Penzen J. Dynamics of structures. Computers \& Structures, pp. 419-422, 1995. 